\title{
A SPATIAL LIGHT MODULATOR USING BSO CRYSTALS
}

\author{
F HOLLER and H J TIZIANI \\ Instıtut fur Technische Optik, Universitat Stuttgart, Pfaffenwaldring 9, D-7000 Stuttgart 80, Fed Rep Germany
}

Received 24 October 1985, revised manuscript received 23 December 1985

\begin{abstract}
The combined use of the photoelectric and electro-optic properties of the $\mathrm{Bi}_{12} \mathrm{SiO}_{20}$ (BSO)-crystal leads to a new possibility of realızing a spatial light modulator Under certan geometncal conditions, which will be discussed, it is possible to realıze, that the BSO-crystal becomes birefnngent depending on the local llluminance, the physical connection between the distributions of birefringence and illuminance will be shown The BSO-crystal combined with a polarizer setup works as a spatial light modulator or as an incoherent to coherent converter
\end{abstract}

\section{Introduction}

To avold speckling and other phase disturbences in coherent optical image processing it is recommendable to use a two dimensional incoherent to coherent (IC)-converter workıng in real time A spatial light modulator (SLM) working in real tıme, which converts lliuminance into transparence, works as such an IC-converter

Most of the currently exısting spatial light modulators work according to the following principle A photoconductive material modulates an applied electric field (or alternating field) along the optical axes in dependence on the local (incoherent) illuminance. Another electrooptic material converts the modulation of the applied electric field into a modulation of birefrnngence A suitable polarizer setup converts the modulation of birefringence into a modulation of transmission The complete optical setup therefore works as an incoherent to coherent (IC)-converter

Some SLM's use liquid crystals as the electrooptic material and $\mathrm{CdS}[1,2]$ or $\mathrm{B1}_{12} \mathrm{SiO}_{20}$ (BSO) - crystals [3] as the photoconductive material, they are rather expensive and their response time is not satisfying Other concepts working with two materials are the microchannel spatial light modulator (MSLM) [4] and the DKDP-converter $[5,6]$ The limit of spatial resolution of the MSLM and the response time of the DKDP-converter do not satisfy the need for most of the applications
Several concepts of holographic IC-conversion have been realized up to now The deformable surface spatial light modulator [7] is limited to 10 lines $/ \mathrm{mm}$ spatial resolution and works with two materials

Another principle of holographic IC-conversion [8] requires only one material, which is able to store a holographic grating in real time such as the BSOcrystal The intensity of the incoherent llumination deforms the grating accordingly The main disadvantage of the holographic IC-conversion is the necessity of a holographic optical setup in order to write the grating

Our aim therefore was to find a material working as well photoconductively electrooptically. Bulk monocrystalline BSO is a suitable material [9-11]. If the optical axis and the applied electric field are mutually perpendicular, the crystal will become birefrungent depending on the local ulluminance $[12,13]$

\section{Spatial light modulation in BSO-crystals}

Physical mechanisms for light pattern recording and erasure in BSO are drift and trapping of photo. electrons under tlumination by incoherent (or coherent) light in the transverse electrooptic configuration This gives rise to a space charge field modulating the refractive index via the linear electrooptic effect [14] Erasure can be achueved by uniform illumination of the crystal

0 030-4018/86/\$03 50 @ Elsever Science Publishers B.V (North-Holland Physics Publishing Division) 


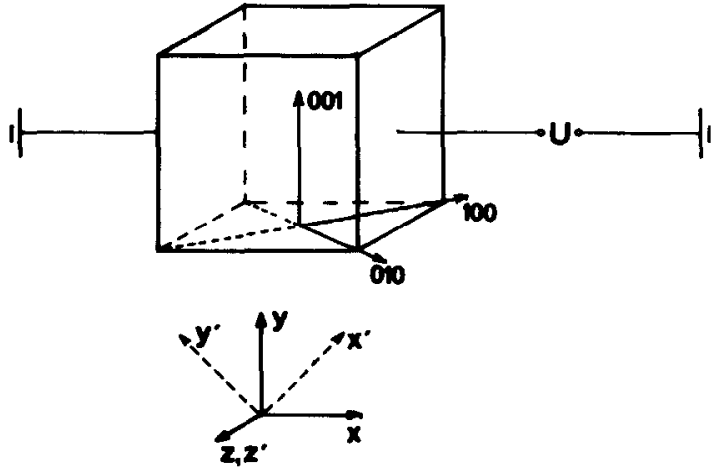

Fug. 1 Crystal orientation (100), (010), (001) crystal axes, $z$ optical axis of image formation, $x$ drrection of the extant electric field, $x^{\prime}, y^{\prime}, z^{\prime}$ optical axes of the crystal

The modulation of the refractive index by the space charge field $E_{\mathrm{sc}}$ is described by the following equation

$n=n_{0}-05 n_{0}^{3} r_{41} E_{\mathrm{sc}}$,

$r_{41}=5 \times 10^{-7} \mathrm{~cm} / \mathrm{kV}$ (see ref [11])

Fig. 1 shows the orientation of the crystal's major optical axes Local birefringence resp polarization change is the result of two different physical effects [13]

(1) The linear electrooptic effect leads to an anisotropic spatial modulation of the refractive index, which is described by the following equations (see fig 1)

$n_{x^{\prime}}=n_{0}-0.5 \cos \left(45^{\circ}\right) n_{0}^{3} r_{41} E_{\mathrm{sc}}$,

$n_{y^{\prime}}=n_{0}+05 \cos \left(45^{\circ}\right) n_{0}^{3} r_{41} E_{\text {sc }}$.

(u) The electrogyratory effect, $1 \mathrm{e}$ the dependence of optical activity with the space charge field.

The space charge field induced by the illumination needs to be calculated In the case of a sinusordal grating $I(x)=I_{0}(1+m \cos k x)$ of high intensity $\left(I_{0}>1 \mathrm{~mW} / \mathrm{cm}^{2}\right)$ and low modulation $(m \ll 1)$ the steady state value of the space charge field in the drift dominant recording mode is given in ref [15]

$E_{\mathrm{sc}}(x)=E_{0} m \cos k x$.

For the more general case $0 \leqslant m \leqslant 1$ the crystal response is no longer linear and the space charge field is given by the following formula (15)
$E_{\mathrm{sc}}(x)=E_{0}\left(1-m_{1}^{2}\right)^{1 / 2} /\left(1+m_{1} \cos k x\right)^{1 / 2}$

$m_{1}$ is the reduced modulation given by formula (5) where $n_{D}$ is the carrier concentration in the dark and $\tau g_{0}$ means the carrier concentration under incident illumination $I_{0}$

$m_{1}=m \tau g_{0} /\left(\tau g_{0}+n_{\mathrm{D}}\right)$.

Combining eqs (2) and (4) leads to

$$
\begin{aligned}
n= & \left(\begin{array}{l}
n_{x^{\prime}} \\
n_{y^{\prime}}
\end{array}\right)=\left(\begin{array}{l}
n_{0} \\
n_{0}
\end{array}\right)+\frac{r_{41} \cos 45^{\circ}}{2} n_{0}^{3} \frac{\left(1-m_{1}^{2}\right)^{1 / 2}}{1+m_{1} \cos k x} \\
& \times E_{0}\left(\begin{array}{c}
-1 \\
+1
\end{array}\right)
\end{aligned}
$$

This result shows, that the crystal's birefrungence is modified depending on the local illumination Combined with an appropriate polarizer setup the crystal works as a spatial light modulator or as an incoherent to coherent converter

The physical mechanisms can be summarized as follows The BSO-crystal, a cubic electrooptic crystal, becomes birefringent when a voltage is applied between the (110)-faces for instance If the incident light is plane, polanzed and orientated to bisect the burefringent axes, the light leaving the BSO-crystal will be elliptically polarized. If we project an inco. herent image into the crystal, the light intensity distribution leads to the appropriate absorption, carriers are generated in the light struck areas These photo generated carriers move in the electric field in a direction to neutralize the applied field thereby reducing the electric field and hence the birefrungence. This leads to the spatial light modulator with the appropriate polarizer arrangement.

When the crystal was placed between crossed polarizers combined with a quarter wave plate compensating the natural birefringence [13] of the crystal, it was possible to convert a modulation of birefringence into a modulation of transmission Fig. 2 shows the optical setup used.

By changing the angle between polarizer and analyzer a positive or negative image can be selected Figs 3 and 4 show this clearly. Fig 5 shows the spectrum of an incoherently illuminated grating after ICconversion

The spatial resolution is very often not only lim- 


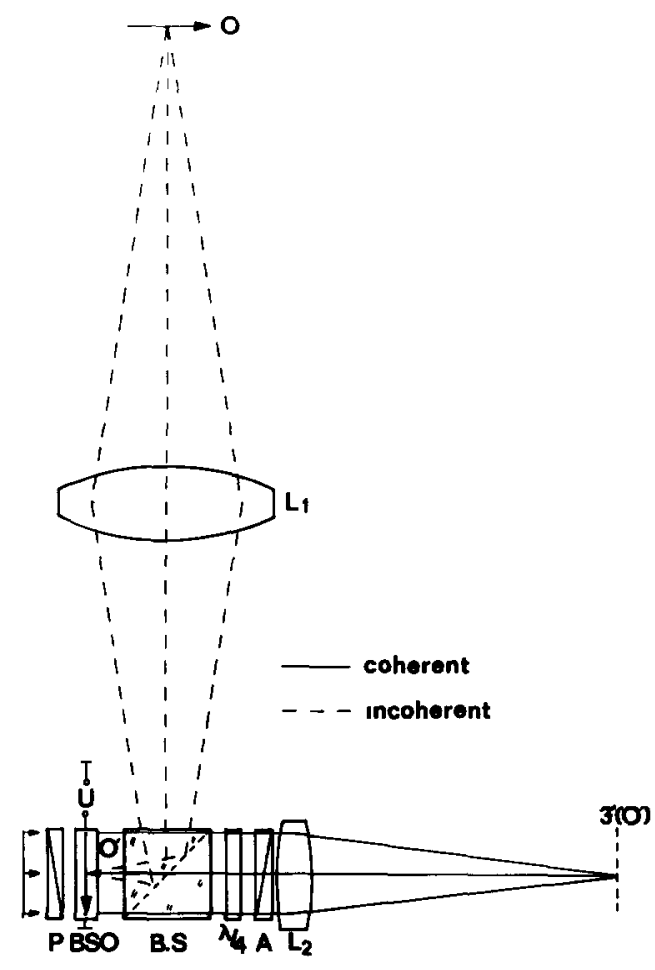

Fig 2 Optical setup used B S Beamsplitter, $\lambda / 4$ quarterwaveplate, $A$ analyzer, $P$ polarizer, $L_{1}$ lens for incoherent image formation, $L_{2}$ Fourier transform lens.

ited by the crystal's properties but also by the crystal's geometry Because of the thickness $d$ of the crystal a slightly defocussed image occurs

The light sensitivity of the crystal depends on the crystal's absorption, namely

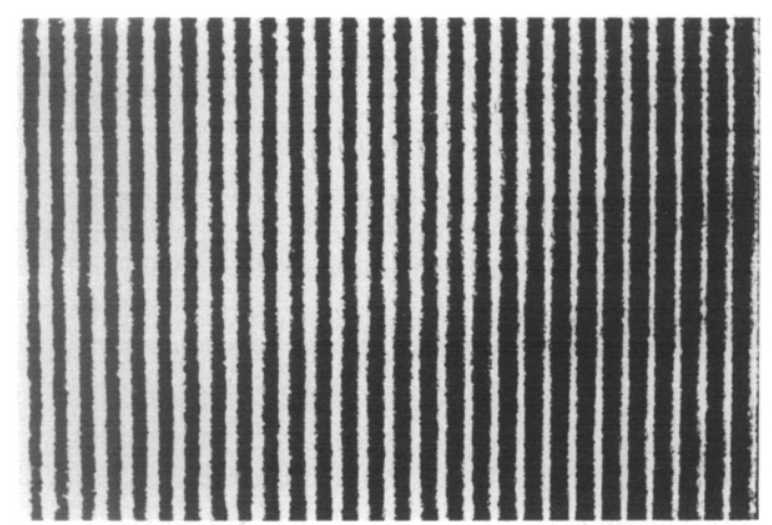

Fig 3. IC-converted picturc of a lattice with a spatial frequency of $10 \mathrm{lnes} / \mathrm{mm}$

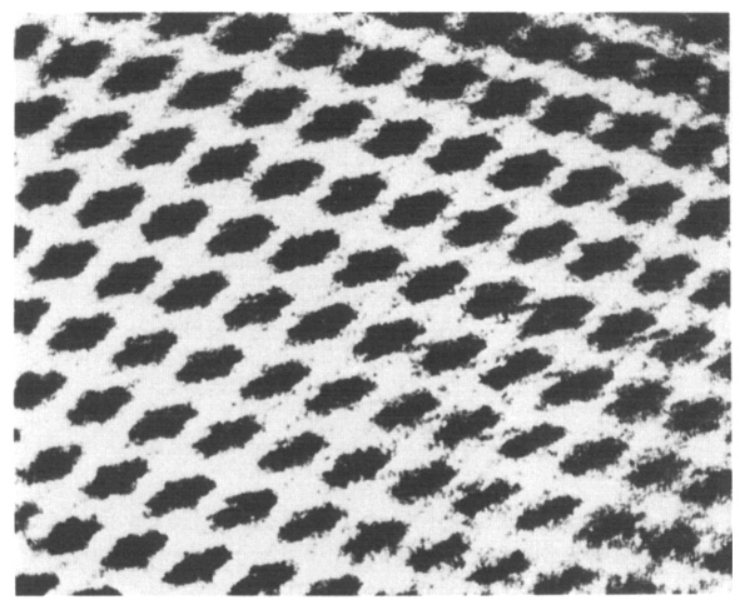

Fig. 4 IC-converted and contrast reversed picture of an electrical shaver's blade

$S=$ const $[1-\exp (-\alpha d)]$

Here $\alpha$ is the absorption coefficient of the crystal Therefore a very thin crystal is not appropriate for our application

The optical transfer function $D(R)$, on the other hand, depends on the aperture ratio, the lateral magnification $\beta$ of the incoherent image formation and the thickness $d$ of the crystal The complete optical transfer function $D_{\mathrm{c}}(R, z)$, where $z$ represents the amount of defocussing, is given by

$D_{\mathrm{c}}(R, z)=D_{\mathrm{BSO}}(R) D_{\mathrm{opt}}(R) D_{\mathrm{def}}(R, z)$

where $D_{\mathrm{BSO}}(R)$ resp $D_{\mathrm{opt}}(R)$ are the OTF's of the BSO-crystal resp the optical system used $D_{\text {def }}(R, z)$ represents the OTF due to defocussing

When reading out the BSO with a plane wave with

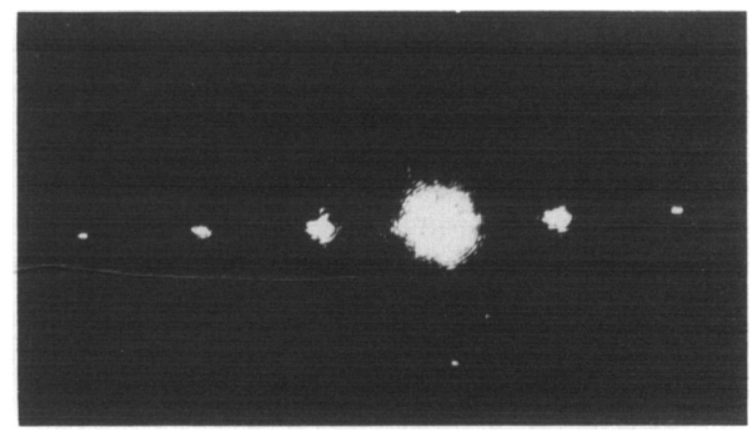

Fig 5 Fourier spectrum of the lattice shown in fig 3 after IC-conversion 


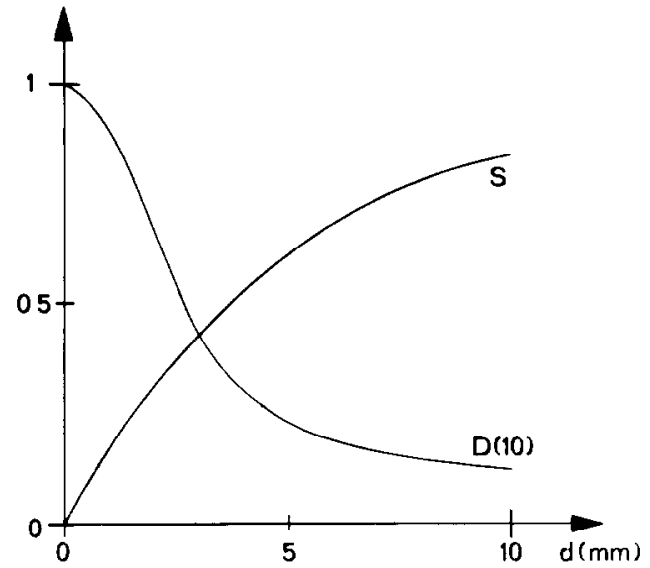

F1g. 6. Modulation transfer function $D(10$ lines $/ \mathrm{mm}$ ) (aperture ratio 14 ) and sensitivity $S$ in dependence on the crystal's thickness $d$

the assumption of small absorption one will get

$D(R)=D_{\mathrm{BSO}}(R) D_{\mathrm{opt}}(R) \frac{2}{d} \int_{0}^{d / 2} D_{\mathrm{def}}(R, z) \mathrm{d} z$

$D_{\text {def }}(R, z)$ is given by the following formula

$D_{\text {def }}(R, z)=J_{1}\left(2 \pi R z \tan \sigma^{\prime}\right) /\left(2 \pi R z \tan \sigma^{\prime}\right)$

$\sigma^{\prime}$ represents the aperture angle in the BSO-crystal Combining formulas (9) and (10) gives

$$
\begin{aligned}
& D(R)=D_{\mathrm{BSO}}(R) D_{\mathrm{opt}}(R)\left(2 / d^{\prime}\right) \\
& \quad \times\left[\left(d^{\prime} / 2\right) J_{0}\left(d^{\prime} / 2\right)+J_{1}(0)-J_{1}\left(d^{\prime} / 2\right)\right. \\
& \left.\quad+\left(d^{\prime} / 2\right) J_{1}\left(d^{\prime} / 2\right) H_{0}\left(d^{\prime} / 2\right)-\left(d^{\prime} / 2\right) J_{0}\left(d^{\prime} / 2\right) H_{1}\left(d^{\prime} / 2\right)\right],
\end{aligned}
$$

where $d^{\prime}=d 2 \pi R \tan \sigma^{\prime}$

For practical applications a compromise between maximum optical transfer function and maximum sensitivity is needed Fig 6 shows a good compromise for $d$ between $1 \mathrm{~mm}$ and $5 \mathrm{~mm}$ thickness Our exper1ments were carried out with crystals of a thickness of $27 \mathrm{~mm}$

\section{Experimental results}

According to eq (6) the electrooptic effect linearly depends on the applied electric field The surrounding atmosphere limits the possible electric field

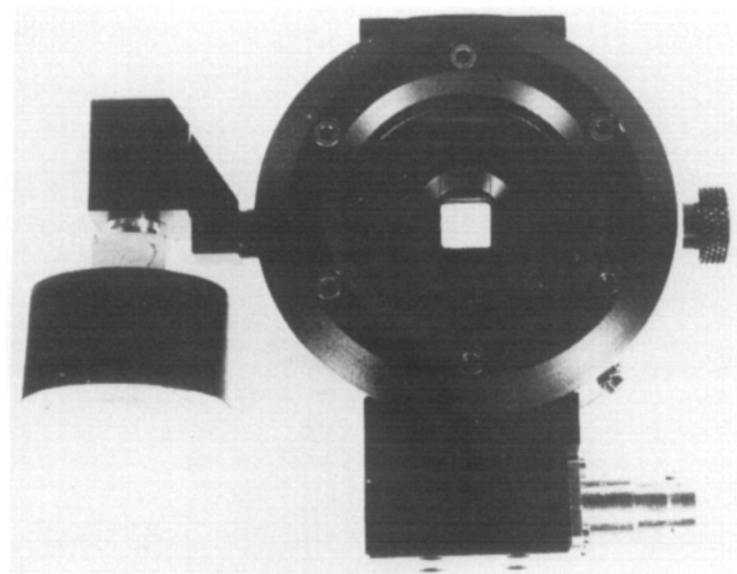

Fig 7 Pressure chamber used The chamber is able to endure 10 bar pressure and more than $10 \mathrm{kV} / \mathrm{cm}$

to about $5 \mathrm{kV} / \mathrm{cm}$, otherwise electrical lightnings could damage the crystal

Because of the small currents (1-10 $\mu \mathrm{A}$ in our experiments) it is not very difficult to produce higher applied fields To avoid crystal damage in this case the crystal was put into a pressure chamber capable to endure a pressure of up to $10 \mathrm{bar}$. Our experiments were carried out with a pressure of 3 bar which made it possible to apply an electric field of $10 \mathrm{kV} / \mathrm{cm}$ for further increase of contrast, fig 7 shows the pressure chamber we used Operation in a vacuum of less than $10 \mu$ bar should be possible too, the technical realization of a pressure of 3 bar seems to be easier.

Figs 8 and 9 show the difference in modulation caused by different applied electric fields $E_{0}$ (see formula (6))

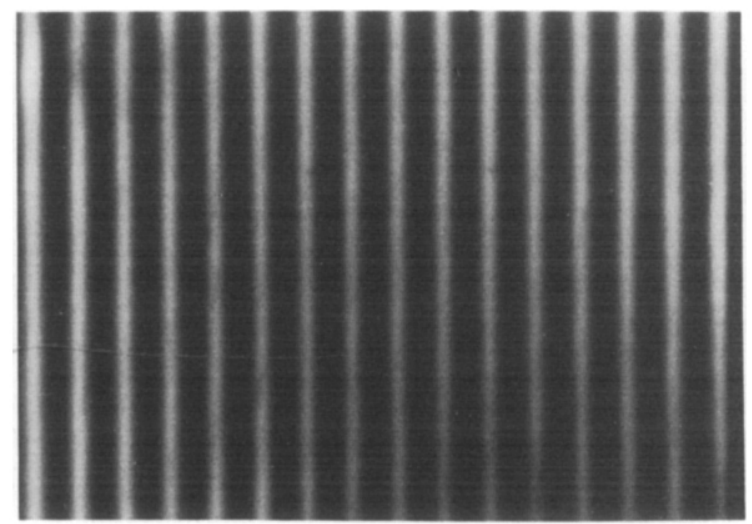

F1g 8 Stored p1cture of a lattice Extant electric freld 10 $\mathrm{kV} / \mathrm{cm}$ 


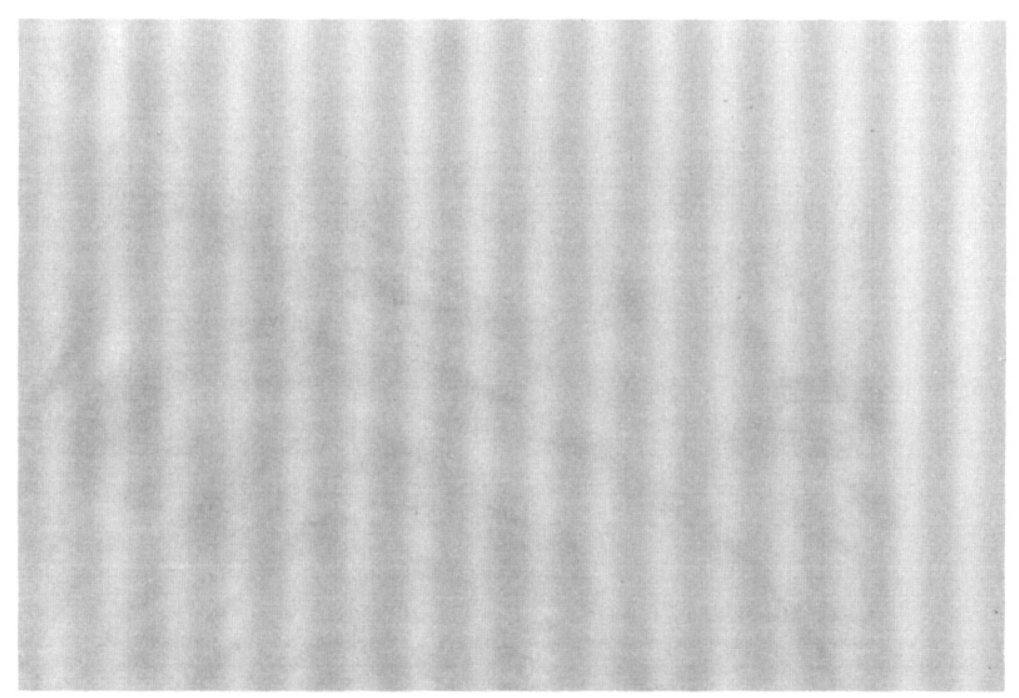

Fig. 9 Stored picture of a lattice. Except for the size of the extant electric field $(2 \mathrm{kV} / \mathrm{cm}$ instead of $10 \mathrm{kV} / \mathrm{cm})$ all $\mathrm{physical}$ conditions are the same as in fig. 8

The response time manly depends on the llum1nance, the applied electric field, and on the wavelength of the light By llluminating the crystal with an electronic flash we achieved a response tıme of about $1 \mathrm{~ms}$

The crystal is sensitive mainly to blue-green light When using the crystal as an incoherent to coherent converter this is an advantage. The information can be stored by using blue or green light and can be read out with red or infrared light without erasure of the information

\section{Characterization}

The new BSO-spatial light modulator is characterized by

- High spatial resolution mainly limited by geometrical optics due to the thickness of the crystal.

- Response time 100-1 ms, depending mainly on the illuminance.

- The application of a pressure chamber allows the use of high electric fields without the risk of damaging the crystal.

- Maximum contrast is limited by the relation be. tween dark- and photocurrent.

- One material, which is as well photoconductive as electrooptic

- Contrast can be easily reversed.

\section{References}

[1] E Marom and J Grmberg, Appl. Optics 16 (1977) 3086

[2] W.P. Bleha, L.T. Lipton, E. Wiener-Avnear, J Grinberg, P.G Rerf, D. Casasent, H.B Brown and B V. Markevitch, Opt Eng. 17 (1978) 371.

[3] P. Auborg, J.P. Hugnard, M. Hareng and R.A. Mullen, Appl. Optics 21 (1982) 3706

[4] C. Warde, A.D Fisher, D.M. Cocco and M.Y. Burmawi, Optıcs Lett. 3 (1978) 196

[5] D. Casasent, Opt. Eng. 17 (1978) 344.

[6] D Casasent, Opt. Eng. 17 (1978) 365.

[7] K Hess and R. Dandliker, Deformable surface spattal lught modulator, Contribution to Horzons de l'Optique 85 , Besançon.

[8] Y Shi, D. Psaltıs, A Marrakch1 and A.R. Tanguay, Appl. Optics 22 (1983) 3665.

[9] A Feldman, W.S Brower and D Horowitz, Appl. Phys Lett 16 (1970) 201.

[10] J.P. Huignard and F. Micheron, Appl. Phys. Lett. 29 (1976) 591.

[11] M. Peltier and F Micheron, J. Appl. Phys. 48 (1977) 3863

[12] W.G. Cady, Prezoelectricity, Vol 2 (Dover Publ, New York, 1964)

[13] J P. Herriau, J. P. Hugnard and P Auborg, Appl. Optics 17 (1978) 1851.

[14] H.J Tzzian, $K$ Leonhardt and J Klenk, Optıcs Comm 34 (1980) 327.

[15] J.P Hugnard and B Ledu, Optics Lett. 7 (1982) 310. 